\title{
La formación en la diversidad y con la diversidad: una necesidad para la formación ciudadana de la población con limitación visual
}

The formation regarding diversity and with diversity: There is a need for the citizen training for people visually impaired

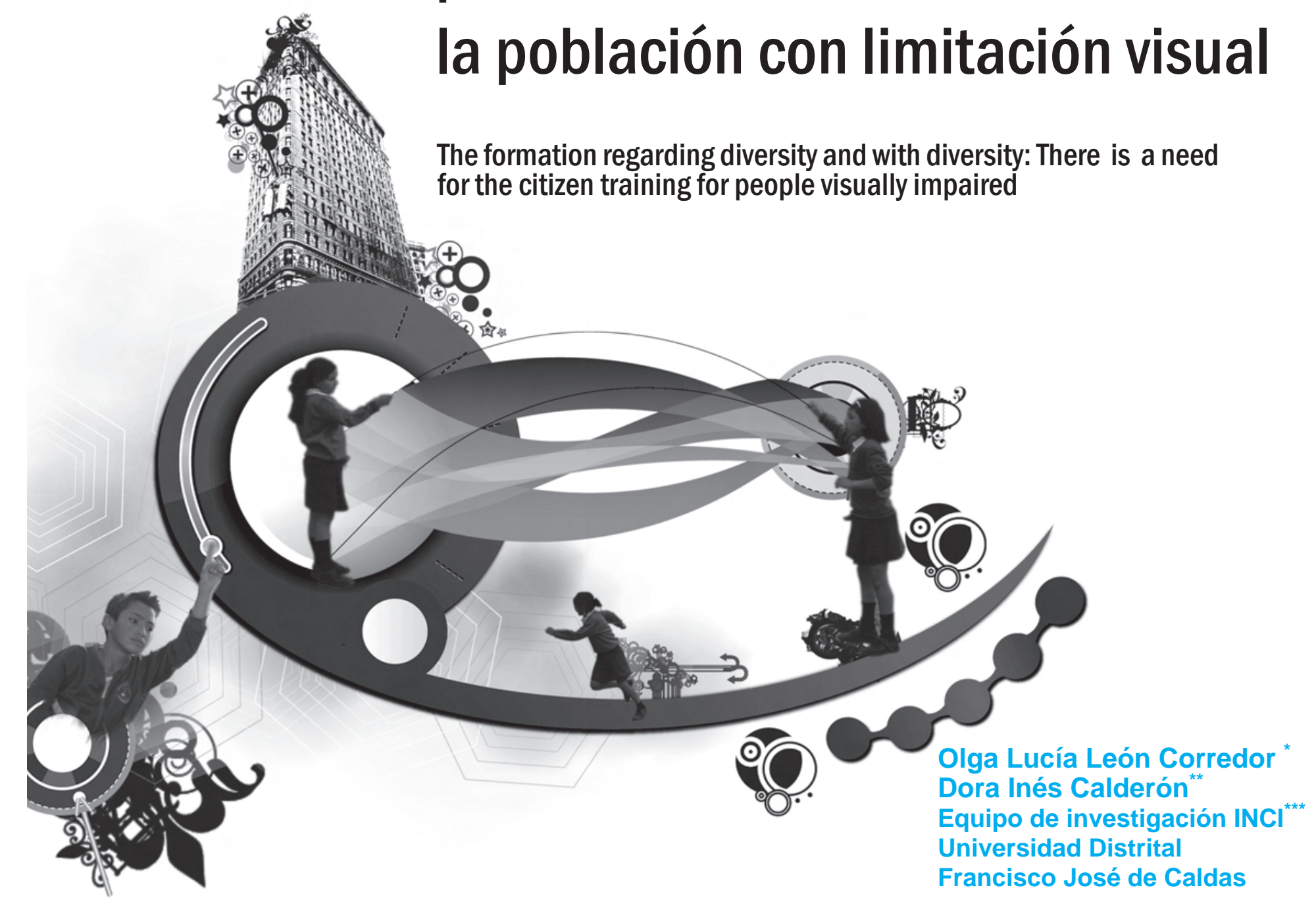

* Docente, Universidad Distrital Francisco José de Caldas. Doctorado en Educación. Correo electrónico: olleon@udistrital.edu.co.
** Docente, Universidad Distrital Francisco José de Caldas. Doctorado en Educación. Correo electrónico: dicalderon@udistrital.edu.co.
*** El equipo INCI estuvo compuesto por Marcela del Castillo, Gladys Sánchez, Gloria Peña, Santiago Rodríguez y Sandra Cortés. 
Resumen Desde una perspectiva de la formación en la diversidad y con la diversidad, se presentan los resultados de la investigación desarrollada por el equipo interinstitucional e interdisciplinar, conformado por investigadores de la Universidad Distrital Francisco José de Caldas y del Instituto Nacional para ciegos (INCI), mediante un convenio de cooperación académica, para indagar la "incidencia de las representaciones sociales sobre la ceguera en el acceso de la población con limitación visual a la educación básica primaria”. Desde una estrategia de investigación etnográfica basada en la recolección de relatos de actores, se contó con la participación de directivos, secretarías de educación, rectores, coordinadores; docentes de área, de grado y de nivel de los niños con limitación visual; los niños con sus familias, las secretarias, los vigilantes, de las seis instituciones educativas de las tres regiones objeto del proceso. De igual manera, se contó con la colaboración de personas con limitación visual no escolarizadas, y sus familias.

\section{Palabras claves: formación, diversidad, población con limitación visual, representaciones sociales, ceguera.}

Abstract From a perspective point of view regarding the formation of diversity and with diversity, here are presented the research results carried out by the interdisciplinary and interinstitutional team conformed by researchers from the "Universidad Distrital Francisco José de Caldas" and the National Institute for the Blind (INCI) through an agreement of academic cooperation, in order to investigate the "incidence of the social representations of blindness regarding the access of visually impaired people to basic primary education”. From an ethnographic research strategy, based on actors collection stories, having in mine the involvement of managers, education secretaries, principals, coordinators, course and area tutors, and of that of children of visually impaired level, children with their families, secretaries, caretakers, of the whole of the six educational institutions in the three aim regions covered in the process. Equally, there were collaboration of visually impaired people that were not at school, and their families.

Keywords: formation, diversity, population with visual discapacity, social representative, blindness 


\section{Introducción}

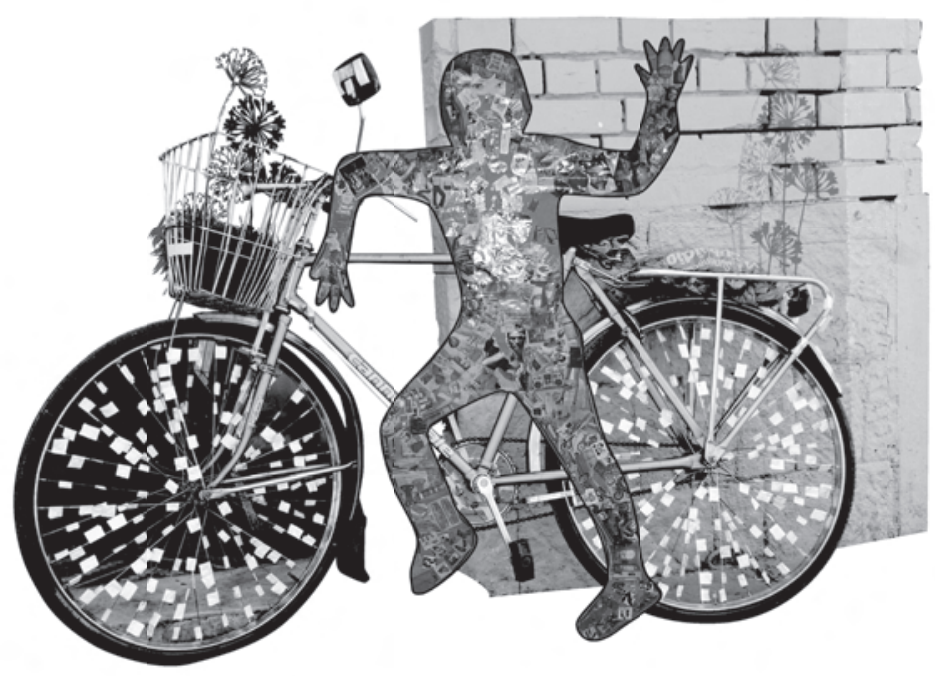

Proponemos un desarrollo a la temática diversidad e inclusión en una doble dimensión: de una parte, consideramos la diversidad de formas de acceso al conocimiento, la diversidad de conocimientos necesarios para el desarrollo social, la diversidad de poblaciones objeto de la educación, y la necesaria articulación de estos tipos de diversidad para posibilitar las condiciones de acceso y permanencia a la institución educativa como espacio físico, de desarrollo social y de formación ciudadana, y como espacio de prácticas culturales de tipo escolar. De otra parte, hemos observado que la diversidad de estructuras presentes en las instituciones educativas y, en particular, en las llamadas "de integración", posibilitan la coexistencia de estrategias de formación de los estudiantes, y la de relaciones entre diversas formas culturales.
Desde el punto de vista anterior, el ingreso de estudiantes con limitación visual a la escuela, en el marco de las políticas de integración educativa, nos lleva a hacer múltiples lecturas de este acontecimiento. Como lo plantea Caicedo (2002), el término "integrar" responde como verbo a una acción que puede entenderse de múltiples maneras, dependiendo del contexto en el que se desarrolle. El escenario para la integración educativa de la población con limitación visual está referido a la escuela integradora; si entendemos que la institución educativa es una organización social que realiza su proyecto de variadas formas, las acciones que desde allí promueve responden de igual manera a esa perspectiva. Es decir, como lo plantea Caicedo (2001), "integrar” es una acción que adquiere forma según la institución que la hace existir; lo que sucede al interior de la escuela está atravesado por intereses, necesidades, contextos, y por los sujetos involucrados en el proceso. 


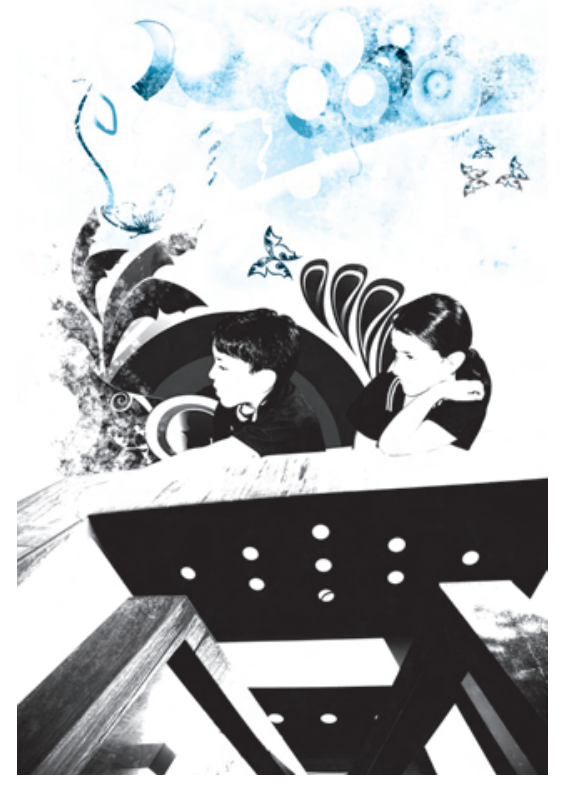

En el contexto específico de la escolarización de estudiantes con limitación visual, encontramos, como lo destacan Ruiz y Azula (2004), la fractura escolar que genera la diversidad cuando ella es considerada como algo impuesto a la escuela y no como una necesidad de crecimiento personal y social de toda la comunidad. De acuerdo con el planteamiento anterior, la escuela y la comunidad educativa tendrían que redefinir su quehacer, sus alcances, sus intereses y sus deseos. Tendría que disminuir el interés por la persona ciega o con baja visión desde su condición particular, como posibilidad de ser integrado, e incrementar el interés por una escuela que construya su proyecto a partir de unos estudiantes con necesidades diferentes, donde estén incluidas las personas ciegas y con baja visión.

\section{La investigación: una alternativa para la comprensión de la formación ciudadana del niño ciego}

El proceso de investigación desarrollado por el equipo de investigación indagó, como se señaló anteriormente, por la "incidencia de las representaciones sociales sobre la ceguera en el acceso de la población con limitación visual a la educación básica primaria”. Con este propósito, se consideró que este fenómeno debía ser comprendido más allá de las políticas educativas declaradas en documentos públicos e institucionales que plantean condiciones para el acceso y la permanencia de las poblaciones con limitaciones al sistema educativo y hacen un llamado a la consideración de la diversidad, la interculturalidad y la integración de este tipo de poblaciones. De ahí que se considerara la necesidad de diseñar un tipo de investigación que diera razón de la relación entre representación social sobre la ceguera y acceso escolar.

Así, la etnografía como método para la caracterización de los fenómenos de acceso escolar de las poblaciones con limitación visual se convierte, a nuestro juicio, en el instrumento metodológico pertinente para la búsqueda de representaciones sociales sobre la ceguera y su efecto en el acceso escolar de estas poblaciones. Particularmente, optamos por realizar la configuración de elementos organizadores de los escenarios de ocurrencia de los fenómenos indagados, desde una perspectiva discursiva. De ahí que se haya acudido a uno de los métodos biográficos (Vasilachis, 2006), el relato, como fuente principal para la indagación etnográfica. 
Adicionalmente, para la consolidación del diseño se optó por la consideración de dos elementos: los escenarios y los actores, constitutivos del entorno de ocurrencia del fenómeno. El escenario está comprendido en la investigación como el espacio de ocurrencia del fenómeno escolar. Se definieron dos tipos de escenarios: macroescenarios, que designan las zonas del país seleccionadas para la investigación: Bogotá, Meta y Quindío, regiones que también se seleccionaron teniendo en cuenta su compromiso con la oferta educativa para la atención de la población con limitación visual; y microescenarios, que hacen referencia a las instituciones escolares de las regiones seleccionadas y los entornos familiares para el caso de niños no escolarizados.

El actor es la categoría central en la investigación, dado que constituye tanto el sujeto configurador y experimentador de representaciones sociales, como el de los procesos de acceso escolar. Así, el proyecto ha identificado tres tipos de actores: niños ciegos escolarizados o no escolarizados, familias de niños ciegos, personal (profesor, celador, secretaria, coordinador, rector) que recibe niños con limitación visual (LV) e instituciones del sistema educativo involucrado en el acceso a la escolaridad de los niños con LV (funcionarios de cadeles o de secretarías de educación en las regiones). Con el fin de contar con la posibilidad de triangularlos, se recogieron tres clases de datos: relatos de los actores, documentos institucionales y planos de las instituciones.

El aporte de la investigación a la comprensión del fenómeno educativo vinculado a la formación ciudadana de las personas con limitación visual se desarrolla en la presentación de los siguientes grupos de resultados.

\section{Las representaciones sociales y la formación} escolar

Nos atenemos aquí a la noción más difundida sobre lo que es una representación social (RS) tomando como fundamento a Serge Moscovici $(1986,1988)$ y a Jean Claude Abric (2001) ${ }^{1}$. Toda representación es una forma de visión global y unitaria de un objeto, pero también de un sujeto. Ella reestructura la realidad y a la vez permite la integración de características objetivas del objeto, de las experiencias anteriores del sujeto y del sistema de normas y actitudes. Según Moscovici, como gran categoría de análisis, la RS atañe a las relaciones que sostienen entre sí los diversos sistemas: ideológico, cognitivo, social y socioeconómico, material y tecnológico. Por ello tiene una doble lógica: la cognitiva y la social. Las RS son conjuntos sociocognitivos, organizados de formas específicas y con reglas propias de funcionamiento. En la génesis de las RS como categoría de análisis está el interés por el estudio de lo colectivo y por las reglas que rigen el pensamiento social. Por ello, en su marco de intereses estaría el estudio de aspectos como el pensamiento ingenuo, el sentido común, la visión del mundo de un grupo social. 
De acuerdo con la fundamentación teórica propuesta para la RS, su componente más importante resulta ser la significación que, a su vez, es determinada por efectos contextuales de dos tipos:

a. Del contexto discursivo, es decir, de la naturaleza de la producción discursiva (contenido, intención y dirección);

b. Del contexto social: lo ideológico y el lugar del individuo en el grupo (jerarquía).

Así pues, el análisis de las RS necesita de una doble identificación: su contenido y su estructura. Toda representación está organizada alrededor de un núcleo central (Heider, 1927, citado por Abric, 2001) y unos elementos periféricos. Según Abric, el núcleo central tiene funciones como las de generar (a partir de él los elementos toman sentido y valor) y organizar (determina la naturaleza de los lazos que unen; unifica y estabiliza la representación). Los elementos periféricos de la representación son conformados por los juicios, las informaciones retenidas, seleccionadas e interpretadas, los estereotipos y las creencias. Estos elementos ilustran, aclaran y justifican la significación. Para Flament (1989, citado por Abric, 2001) los elementos periféricos constituyen esquemas organizados por el núcleo central. Por esta razón, cumplen funciones prescriptoras de los comportamientos y de la toma de posiciones y permiten una modulación personalizada de las representaciones y de las conductas asociadas.
En el análisis de RS consideramos, prioritariamente, dos elementos articuladores, el grupo social, y en él el sujeto, como los actores que experimentan y que construyen RS; y el relato, como el escenario discursivo en el que se manifiestan tales RS.

Se consideró la escuela como una de las instituciones sociales en la que se desarrollan prácticas de socialización de los sujetos, en este caso, de tipo escolar. Estas prácticas educativas constituyen el escenario de configuración de los sujetos desde la perspectiva de la formación académica que legitimará, más tarde, otras prácticas profesionales, científicas, tecnológicas, artísticas, etc., fundamentales para el desarrollo de las potencialidades de los sujetos y de la cultura en general. 
En el marco anterior, la investigación se sitúa en el interés por el sujeto con limitación visual (SLV) desde su relación con los procesos de escolarización. Se indaga por fenómenos relacionados con las subjetividades y las intersubjetividades y, en ellas, la configuración de sentido y de significación social en su proceso de acceso y permanencia a la escolaridad primaria, en tanto un derecho sociocultural del ser humano. Desde este punto de vista, el desentrañamiento de las representaciones sociales y la identificación de fenómenos de escolaridad exigió una postura que partió de reconocer la ceguera como una condición de desarrollo social.

\section{Las representaciones sociales sobre la} ceguera: entre la integración y la incapacidad

En términos de discusión, el procedimiento analítico para la identificación de representaciones sociales permitió una metodología para la identificación del modo como se configuraron las representaciones. Presentamos una síntesis de los hallazgos sobre representaciones sociales sobre la ceguera, considerando que, en general, se hallaron dos grandes marcos de representaciones sociales sobre la ceguera: uno relacionado con la condición de "ser ciego", y otro, con "la posibilidad educativa del estudiante con limitación visual".

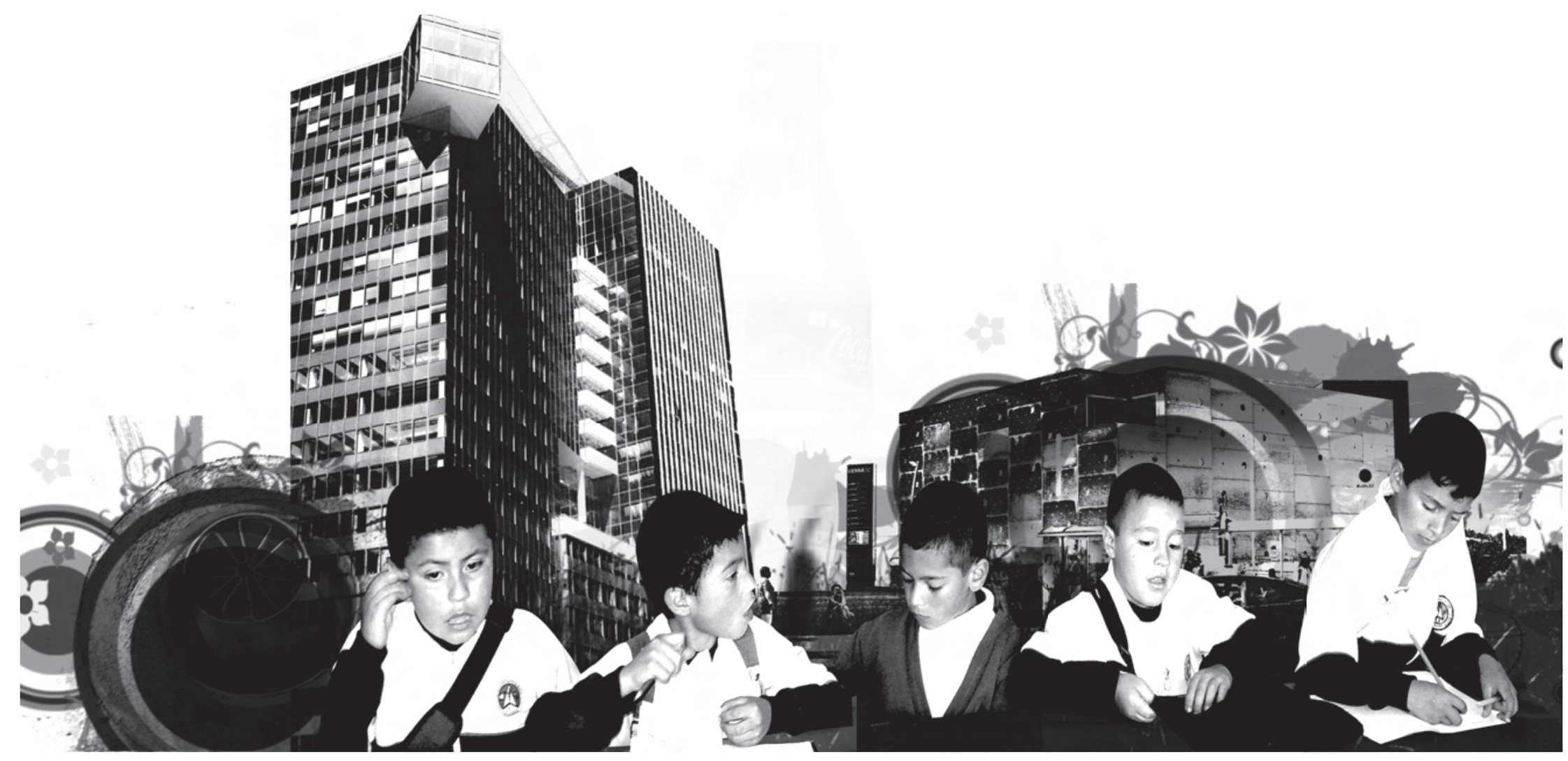


Tabla 1. Representaciones sobre la condición de ser ciego

\begin{tabular}{|c|c|c|}
\hline $\begin{array}{l}\text { Núcleos de } \\
\text { representaciones }\end{array}$ & Elementos periféricos de soporte & $\begin{array}{c}\text { Actores } \\
\text { vinculados }\end{array}$ \\
\hline $\begin{array}{l}\text { La ceguera es } \\
\text { anormalidad }\end{array}$ & $\begin{array}{l}\text { Es algo de cuidado. En los discursos se presenta la alusión a "normalidad y } \\
\text { "anormalidad" para referirse a la situación del niño ciego frente a los demás. } \\
\text { Se acuña una forma de hablar que reitera enunciados como "yo creo que es } \\
\text { normal", "lo trato como si fuera normal", lo cual revela que se toma la ceguera } \\
\text { como discapacidad. Se muestra prevención frente a ella. Se plantea que "no es } \\
\text { tan fácil integrar". Se acepta que hay momentos en que se desconoce la presencia } \\
\text { del estudiante limitado visual. La presencia del niño ciego en el aula aún es algo } \\
\text { contingente, extraordinario... genera sentimientos de temor y prácticas de } \\
\text { indiferencia. }\end{array}$ & $\begin{array}{l}\text { - Docentes } \\
\text { - Coordinadores } \\
\text { - Padres }\end{array}$ \\
\hline $\begin{array}{l}\text { El ciego es } \\
\text { dependiente }\end{array}$ & $\begin{array}{l}\text { Para su desplazamiento requiere de la compañía de alguien. En sus quehaceres } \\
\text { escolares depende de su familia o del maestro de apoyo y en sus relaciones sociales } \\
\text { en la escuela está supeditado también al maestro de apoyo, a "un compañero" que } \\
\text { se le dedique. De lo contrario no participa, se queda solo. Genera sentimientos de } \\
\text { compasión y prácticas de solidaridad o de indiferencia. }\end{array}$ & $\begin{array}{l}\text { Niño } \\
\text { escolarizado } \\
\text { Madre } \\
\text { Docentes } \\
\text { titulares y } \\
\text { de apoyo }\end{array}$ \\
\hline $\begin{array}{l}\text { El ciego es } \\
\text { incapaz }\end{array}$ & $\begin{array}{l}\text { El ciego no puede hacer lo que hacen los demás... Este rasgo se encuentra unido } \\
\text { con la admiración, pero al momento de evaluar los docentes plantean formas } \\
\text { "menos exigentes" y en algunos casos sin criterio concreto de evaluación. Se } \\
\text { excluye al invidente de tareas escolares que se consideran imposibles o difíciles } \\
\text { para él; se reemplazan por algo que "sí pueda hacer". Sin embargo, se le califica } \\
\text { con notas sobresalientes. Genera sentimientos de compasión y de baja autoestima } \\
\text { y de frustración para el niño ciego y prácticas de exclusión socioescolar y cultural } \\
\text { y de traslado de responsabilidad (al docente de apoyo, a la familia). }\end{array}$ & $\begin{array}{l}\text { Docente } \\
\text { titular }\end{array}$ \\
\hline
\end{tabular}

Aunque es posible considerar límites débiles entre cada núcleo central identificado y se pudiera concluir que la idea de "anormalidad" es el gran núcleo central y que los otros dos son periféricos de éste, establecimos las diferencias por cuanto cada uno de los núcleos reveló consideraciones periféricas que permiten establecer, en particular, discursos y prácticas diferentes. Adicionalmente, es posible inferir que este conjunto de representaciones obedece prioritariamente a factores de construcción cultural e histórica de creencias y de imaginarios sociales; es decir, encontramos en este primer conjunto representacional la huella cultural de la representación y quizás la más arraigada en la colectividad. 
Tabla 2. Representaciones sobre la posibilidad educativa del niño ciego

\begin{tabular}{|c|c|c|}
\hline $\begin{array}{l}\text { Núcleos de } \\
\text { representaciones }\end{array}$ & $\begin{array}{l}\text { Síntesis de elementos } \\
\text { periféricos de soporte }\end{array}$ & $\begin{array}{c}\text { Actores } \\
\text { vinculados }\end{array}$ \\
\hline $\begin{array}{l}\text { El ciego necesita } \\
\text { educación especial }\end{array}$ & $\begin{array}{l}\text { La formación escolar del estudiante ciego está a cargo, } \\
\text { primordialmente, del docente de apoyo, quien termina } \\
\text { convirtiéndose en un "sabedor" de todas las asignaturas, en el } \\
\text { conocedor de los instrumentos de apoyo y en el soporte del profesor. } \\
\text { Genera sentimientos de solidaridad entre profesores y prácticas de } \\
\text { cooperación o de traslado de responsabilidad educativa al docente de } \\
\text { apoyo o a la familia, inclusive, en Bogotá, al CRAC }{ }^{2} \text {. }\end{array}$ & $\begin{array}{l}\text { - Docentes: titulares y } \\
\text { de apoyo } \\
\text { - Coordinadores } \\
\text { - Padres de familia }\end{array}$ \\
\hline $\begin{array}{l}\text { El ciego se } \\
\text { puede "integrar" }\end{array}$ & $\begin{array}{l}\text { Se jalona desde el discurso sobre integración educativa. Es elocuente } \\
\text { y positivo. Se explicitan las bondades de la política de integración. } \\
\text { Es un discurso instalado desde la oficialidad. Genera sentimientos } \\
\text { de orgullo y de identidad, en otros casos de obligatoriedad, por } \\
\text { ser partícipe de la ejecución de la política y prácticas de gestión } \\
\text { administrativa. }\end{array}$ & $\begin{array}{l}\text { - Secretarios de educación } \\
\text { - Rectores } \\
\text { - Coordinadores } \\
\text { - Profesores }\end{array}$ \\
\hline $\begin{array}{l}\text { El ciego } \\
\text { "es de admirar" }\end{array}$ & $\begin{array}{l}\text { Los docentes reconocen que los niños ciegos tienen muchas } \\
\text { capacidades y los catalogan como supremamente inteligentes, } \\
\text { de buena memoria y muy juiciosos. Dentro del espectro de los } \\
\text { sentimientos se halla admiración del personal administrativo hacia } \\
\text { los estudiantes con limitación visual y reconocimiento del personal } \\
\text { docente. Se generan prácticas de solidaridad. }\end{array}$ & $\begin{array}{l}\text { - Docentes de apoyo } \\
\text { - Docentes titulares } \\
\text { - Coordinadores } \\
\text { - Rectores } \\
\text { - Vigilantes }\end{array}$ \\
\hline
\end{tabular}

En este conjunto de representaciones, que no están desligadas de las primeras, se observan las que surgen a partir de políticas educativas de "integración e inclusión" 3 para poblaciones vulnerables en Colombia. Desde este punto de vista, son las más débiles, que están en construcción y que por ello también pueden variar si el actor cambia de papel. Se trata más bien de las que soportan los discursos oficiales de la educación para las poblaciones con discapacidades o necesidades educativas especiales.

2 En Bogotá el CRAC es el Centro de Rehabilitación para Adultos Ciegos.

3 No discutiremos en este artículo la relación entre integración e inclusión, dado que no es el propósito de la investigación. Tan sólo se refiere el hecho de ser la vía política por la que se plantea el acceso a la educación básica de la población invidente. 
Por otra parte, como grandes resultados de representaciones sociales sobre la ceguera, se identifican dos, según campos de significaciones constituidos en los relatos:

La ceguera-integración. Se construye a partir del discurso proveniente de las políticas de integración de la población con limitación visual (LV) a las aulas regulares y se posiciona, como configuración semántica, en los discursos y en las prácticas de los diversos actores, en especial de los directivos, los coordinadores y los profesores. Se observa que los aspectos relacionados con la integración, como la expresión de políticas y de creencias y actitudes frente a ellas, ha constituido el “campo de la integración”, y posicionado una exigencia que ha de ser cumplida por parte de los actores, más que una actitud arraigada en la representación sobre la ceguera, esto, más allá del compromiso de directivos, funcionarios y maestros a quienes atañe directamente la política. El conocimiento de ésta implica la necesaria elaboración discursiva de una forma de hablar sobre la limitación visual y la escuela; sin embargo, las prácticas socioacadémicas escolares presentan distancia y dificultad para hacer compatible la representación ceguera-integración manifiesta prioritariamente en el discurso de dichas políticas. Puede decirse, desde esta perspectiva, que la representación ceguera-integración se encuentra en una fase de constitución, como efecto de un discurso vertical de política pública. Veamos fragmentos de uno de los relatos en donde se identifica este tipo de RS.
La ceguera-anormalidad. El segundo gran núcleo de significación identificado, ceguera-anormalidad y ceguera-temor, proviene de factores socioculturales e históricos. Estas representaciones se explicitan en los discursos referidos a las valoraciones sobre la situación de "estar ciego" (bien como autocondición o como la condición del hijo o del estudiante). Sin embargo, también se ocultan en el contradiscurso de la admiración, de la compasión y de la solidaridad. A pesar de las manifestaciones de optimismo y de admiración por los niños ciegos, se insinúa en el discurso de los actores el sentimiento de "lástima” y de "pesar" por la condición de ceguera y, con ello, la esperanza de que esta condición se "solucione". Se requeriría una investigación más larga que permitiera observar con profundidad prácticas, creencias, imaginarios, sobre la ceguera como fenómeno sociocultural, más allá de la condición física, fisiológica. En general, es posible plantear que esta representación tiene su anclaje en fuertes raíces socioculturales e históricas. Por esta razón, puede llegar a superponerse a la primera, a pesar del esfuerzo de producción del discurso de la política educativa. Adicionalmente, es posible inferir que este conjunto de representaciones identificadas es el resultado de factores de construcción cultural e histórica de creencias y de imaginarios sociales; es decir, encontramos en el primer conjunto la huella cultural de la representación y quizás la más arraigada en la colectividad: la ceguera como una situación discapacitante para la persona con limitación visual. 


\section{Proyecciones de la investigación}

Comprender los sistemas de significación social que orientan las prácticas sociales resulta ineludible para el investigador educativo por cuanto se muestra, una vez más, la profunda relación existente entre modos de pensar, individual y colectivamente, y prácticas sociales. Una vía para el acercamiento a tales significaciones sociales es, sin duda, la identificación de representaciones sociales. Así, para el caso de la educación de las personas con limitación visual, este estudio logró un marco de comprensión de las significaciones que circulan socialmente acerca de la ceguera y del papel que tales significaciones juegan en las interacciones que ocurren en los espacios escolares integrados por niños con limitación visual.

En la investigación de representaciones sociales cobra gran valor la reflexión sobre el método de análisis de RS. Este es uno de los puntos críticos que históricamente se plantean para las investigaciones sobre RS. En ese sentido, esta investigación configuró un modo de análisis a partir de la propuesta clásica de Moscovici (1986) y Abric (2001) sobre la estructura de la RS en núcleo central y elementos periféricos. A nuestro juicio, y desde una perspectiva discursiva, la fecundidad de esta propuesta es grande, por cuanto se configura una propuesta analítica coherente para la interpretación empírica e inferencial de las significaciones a través de los discursos de los actores sociales. Se garantiza, con ello, la explicitación organizada de las significaciones elaboradas por los sujetos que intervienen en los escenarios indagados, restringiendo, de alguna manera, la posibilidad especulativa del investigador.

Se hace necesario, entonces, permanecer cerca del relato, cerca del decir de los actores, y emplear la teoría para corroborar, analizar y contrastar las significaciones reconstruidas. He ahí un modo de investigación que requiere la capacidad de navegar en los discursos y de hallar en ellos los signos de RS particulares, de escuchar atentamente los implícitos, articular con explícitos y armar los núcleos de significación que sostienen las RS.

En cuanto a los resultados obtenidos, a pesar de las relaciones encontradas con la limitación visual, se identificaron dos grandes representaciones sociales que circulan sobre la ceguera en contextos escolares: la RS que relaciona ceguera-integración, que, como se

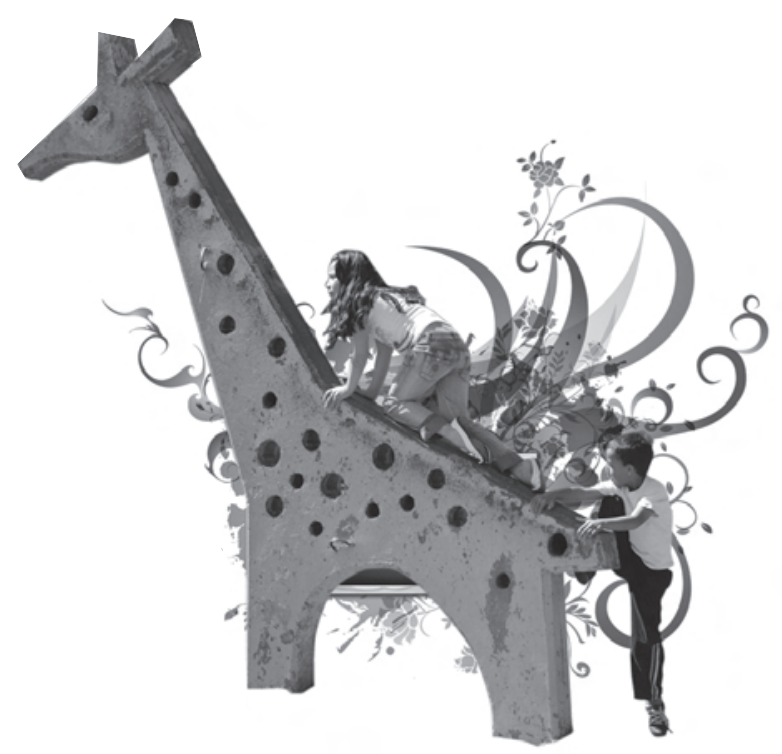




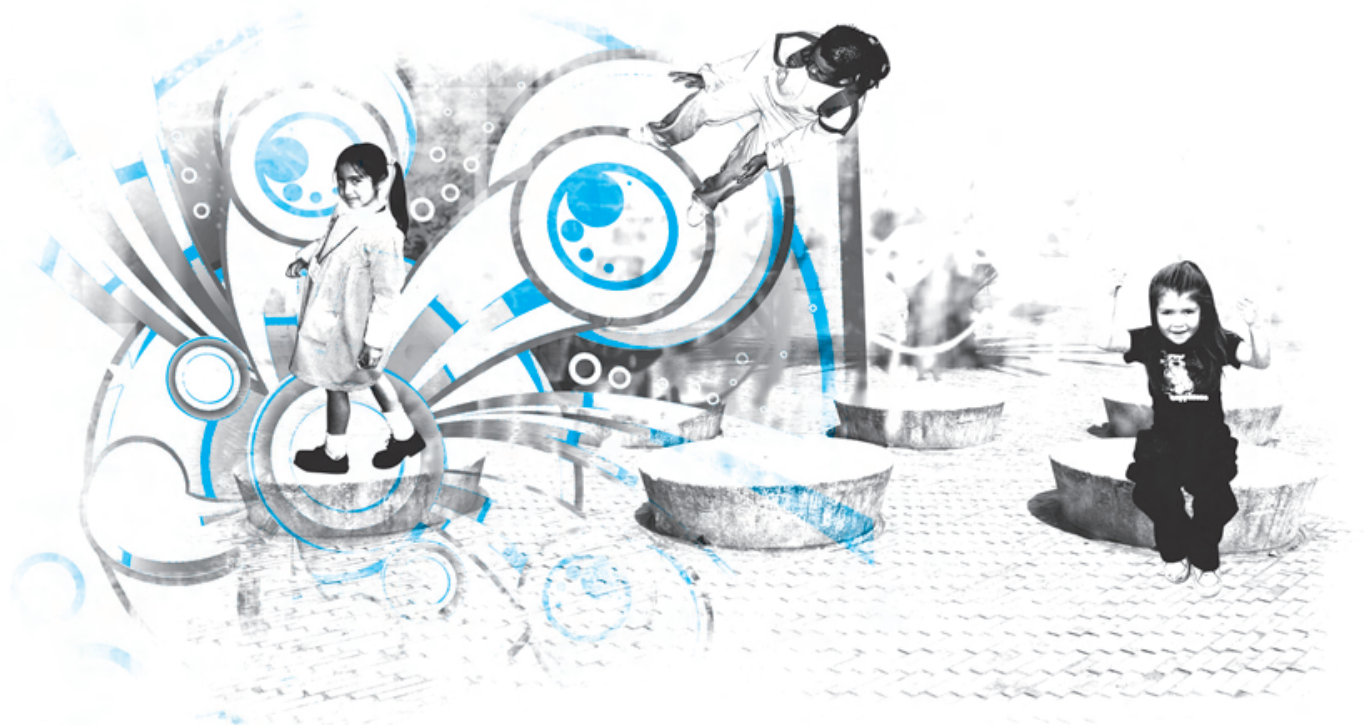

dijo, es una representación en construcción, producto de los discursos políticos sobre la educación de las personas ciegas. Más allá del carácter incipiente de ella, vale la pena considerar, en este momento, que los modos de construcción social de sentido para la expresión "integración” serán determinantes en la consolidación de esta representación, por cuanto el discurso de integración está acompañado de prácticas socioeducativas que necesariamente pasan por el contraste con las creencias y las representaciones históricamente construidas sobre la ceguera, sobre la discapacidad, sobre la diferencia. En consecuencia, será importante para la investigación educativa seguir la pista al desarrollo de esta representación.

Por otra parte, la segunda gran representación identificada es la de la ceguera-anormalidad. Siendo la más arraigada y con mayor tradición histórica, se observa el papel central que juega en las prácticas escolares que rodean a niños ciegos. Como se denota en los relatos y en la identificación de núcleos centrales y de elementos periféricos, las opiniones y prácticas relacionadas muestran cómo a pesar del esfuerzo de los actores por considerar a los limitados visuales como iguales, como capaces, se evidencian sentimientos que oscilan entre la admiración, el pesar, la incredulidad, la esperanza de recuperación de la visión, y la impotencia; esto, acompañado de prácticas de rechazo, de indiferencia, de conmiseración.

Así pues, vale la pena considerar el valor investigativo de la identificación de RS en los contextos escolares y, desde ahí, emprender acciones concretas para impactar la formación social y cultural del niño con limitación visual, desde los ambientes escolares. 


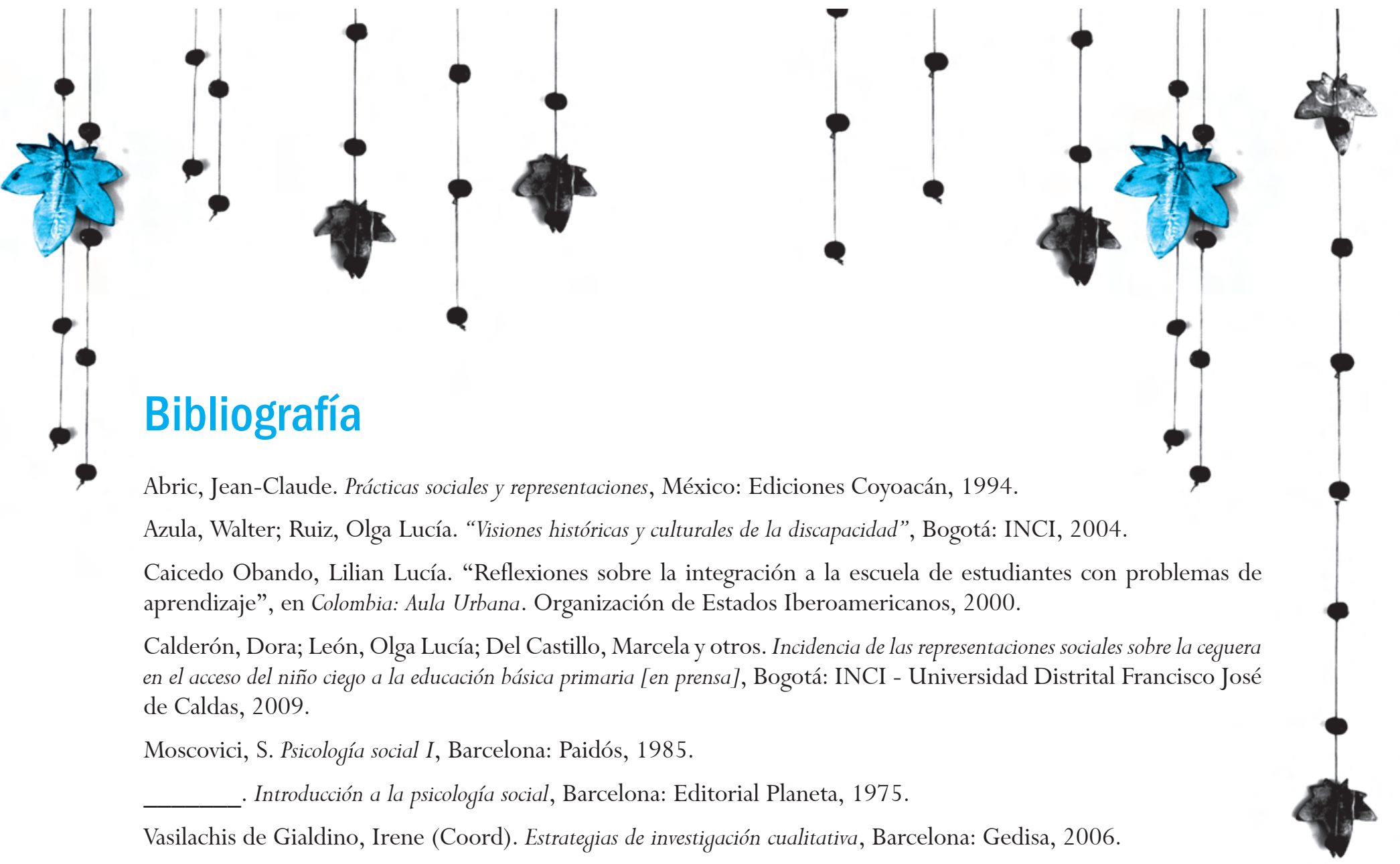

